postmenopausal women (PMW) and age-matched men. Evaluate DA in WoCBA comparing to the aforementioned groups.

Methods: Observational transversal study, using data from the portuguese registry of rheumatic diseases (Reuma.pt) from 3 portuguese centers. Adult patients (pts) with the diagnosis of RA, PsA, AS or JIA were allocated to the following groups: WoCBA (aged 18-44y), young men (YM) (18-44y), PMW ( $\geq 45 y$ ) and matched men $(\geq 45 y)$. Demographic and clinical variables are described as means or frequencies. Differences between groups regarding therapy and DA were assessed with Chi-square and ANOVA tests. Linear and logistic regression models were used to find predictors of DA and prescription patterns.

Results: 2133 pts were included, $69.9 \%$ female with a mean age of $55.96 \pm 15.85$ y. 1437 pts were diagnosed with RA, 305 with PsA, 254 with AS and 137 with JIA. Patterns of prescription are detailed in table 1. WoCBA were less likely to be treated with glucocorticoids than PMW (OR $0.6695 \% \mathrm{Cl} 0.44-0.99$ ). WoCBA were 1.76 times more likely to be treated with MTX than YM $(95 \% \mathrm{Cl} 1.04-2.97)$. Certolizumab was specially prescribed in WoCBA (OR 13.8, 95\%Cl 1.4-132.8). WoCBA had significantly higher DA scores than YM (DAS28 $3.03 \pm 1.39$ vs $2.32 \pm 1.18$ and BASDAI $3.55 \pm 2.0$ vs $2.43 \pm 1.66$ )

Table 1. Prescription patterns

\begin{tabular}{lccccc}
\hline Medications, n (\%) & $\begin{array}{c}\mathrm{A} \text {-WoCBA } \\
(\mathrm{N}=256)\end{array}$ & $\begin{array}{c}\mathrm{B} \text { - Young } \\
\text { Men } \\
(\mathrm{N}=161)\end{array}$ & $\begin{array}{c}\text { C - Post menopau- } \\
\text { sal Women } \\
(\mathrm{N}=927)\end{array}$ & $\begin{array}{c}\mathrm{D}-\text { Men } \\
\mathrm{N}=340\end{array}$ & $\begin{array}{c}\text { Chi-square } \\
\text { test }\end{array}$ \\
\hline NSAIDs & $143(55.9)$ & $111(68.9)$ & $472(50.9)$ & $169(49.7)$ & $\mathrm{p}<0.001$ \\
Glucocorticoids & $106(41.4)$ & $31(19.3)$ & $625(67.4)$ & $154(45.3)$ & $\mathrm{p}<0.001$ \\
csDMARDs & & & & & \\
- Methotrexate & $149(58.2)$ & $60(37.3)$ & $663(71.5)$ & $197(57.9)$ & $\mathrm{p}<0.001$ \\
- Leflunomide & $12(4.7)$ & $4(2.5)$ & $45(4.9)$ & $2(0.6)$ & $\mathrm{p}=0.003$ \\
- Sulfassalazine & $9(3.5)$ & $5(3.1)$ & $39(4.2)$ & $9(2.7)$ & $\mathrm{NS}$ \\
- Hydroxychloroquine & $36(14.1)$ & $4(2.5)$ & $117(12.6)$ & $18(5.3)$ & $\mathrm{p}<0.001$ \\
bDMARDs & & & & & \\
- Etanercept & $48(18.8)$ & $29(18.0)$ & $140(15.1)$ & $66(19.4)$ & $\mathrm{NS}$ \\
- Infliximab & $9(3.5)$ & $11(6.8)$ & $36(3.9)$ & $30(8.8)$ & $\mathrm{p}=0.002$ \\
- Adalimumab & $17(6.6)$ & $15(9.3)$ & $47(5.1)$ & $27(7.9)$ & $\mathrm{NS}$ \\
- Golimumab & $15(5.9)$ & $18(11.2)$ & $37(4.0)$ & $30(8.8)$ & $\mathrm{p}<0.001$ \\
- Certolizumab & $10(3.9)$ & $0(0)$ & $2(0.3)$ & $2(0.6)$ & $\mathrm{p}<0.001$ \\
- Tocilizumab & $12(4.7)$ & $2(1.2)$ & $71(7.7)$ & $10(2.9)$ & $\mathrm{p}<0.001$ \\
- Rituximab & $5(1.9)$ & $0(0)$ & $50(5.4)$ & $4(1.2)$ & $\mathrm{p}<0.001$ \\
- Abatacept & $0(0)$ & $0(0)$ & $9(1)$ & $0(0)$ & $\mathrm{NS}$ \\
- Secukinumab & $1(0.4)$ & $4(3.5)$ & $8(0.9)$ & $2(0.6)$ & $\mathrm{NS}$ \\
- Ustekinumab & $1(0.4)$ & $3(1.9)$ & $5(0.5)$ & $0(0)$ & $\mathrm{NS}$ \\
tsDMARDs & $4(1.6)$ & $1(0.6)$ & $9(0.9)$ & $0(0)$ & $\mathrm{NS}$ \\
\hline
\end{tabular}

DDMARDs - biologic disease modifying antirheumatic drugs, csDMARDs - conventional synthetic disease modifying antirheumatic drugs, NSAIDs - non-steroidal anti-inflammatory drugs, tsDMARD - targeted synthetic disease modifying antirheumatic drugs, WoCBA women of childbearing age

Conclusion: Certolizumab was prescribed preferentially in WoCBA, who alsoreceived more MTX than YM. Nevertheless, DA in this group was not well controlled, which may influence future pregnancy outcomes. Ensuring tight DA control in WoCBA through proper and ideally no teratogenic medication remains an unmet clinical need.

Disclosure of Interests: None declared

DOI: 10.1136/annrheumdis-2020-eular.1976

\section{AB1146 REAL-LIFE PRACTICES IN MANAGEMENT OF REPRODUCTIVE HEALTH IN SLE AND APS BY OBSTETRICIANS AND RHEUMATOLOGISTS IN EGYPT. (AN ONLINE-BASED QUESTIONNAIRE)}

A. Beltagy ${ }^{1}$, N. Eshak ${ }^{2}$, M. Morsy ${ }^{1}$, S. Shoela ${ }^{3}$, F. Fayed ${ }^{4}$, S. Aly ${ }^{5}$, A. Emam ${ }^{6}$, A. El-Girby ${ }^{7} .^{1}$ Alexandria University, Faculty of Medicine, Internal Medicine, Rheumatology Department, Alexandria, Egypt; ${ }^{2}$ Texas Tech University Health Sciences Center, Internal medicine, Lubbock, United States of America; ${ }^{3}$ Alexandria University, Faculty of Medicine, Internal Medicine, Rheumatology Department, Alexandria, Egypt; ${ }^{4}$ Alexandria University, Alexandria Students Hospital, Internal Medicine, Rheumatology, Alexandria, Egypt; ${ }^{5}$ Alexandria University, Alexandria, Egypt; ${ }^{6}$ Alexandria University, Obstetric and Gynecology, Alexandria, Egypt; ${ }^{7}$ Alexandria University, Internal Medicine, Rheumatology, Alexandria, Egypt

Background: Systemic lupus erythematosus (SLE) is an auto-immune disease that affect women in their reproductive age. Antiphospholipid syndrome (APS) is a hypercoagulable immune disease that occur as a primary condition or in assosiation with SLE.

The reproductive aspects as contraception, fertility, pregnancy are crucial to consider for proper management of SLE/APS.
Addressing these issues require collaboration between rheumatologists and obstetricians, improving their knowledge and ensuring that both are acquainted with the updated guidelines.

Objectives: To assess the knowledge and practice of Egyptian obstetricians and rheumatologists in management of reproductive health issues in SLE and APS, and to detect common misconceptions.

Methods: This research was conducted via google form online survey based on points discussed in EULAR recommendations for women's health and the management of family planning, assisted reproduction, pregnancy and menopause in patients with SLE and/or APS. ${ }^{1}$ It was sent to target obstetricians and rheumatologists by internet clouds like (Facebook, twitter, Linkedln) from August to November 2019. It included five domains; demographic data, general knowledge and attitudes about pregnancy in SLE and APS, contraception, drugs, and assisted reproductive techniques (ART)

After submitting answers, respondents were shown a link directing them to the 2016 EULAR recommendations. ${ }^{1}$

Results: This study was conducted on 254 physicians, $62 \%$ obstetricians and $38 \%$ rheumatologists. $64.6 \%$ were between the ages of $30-35$ years.

For general knowledge, $52 \%$ of Obstetricians considered pregnancy in inactive SLE to be risky. ( $79.4 \%$ vs $54.1 \%$ ) of (rheumatologists and obstetricians) respectively test for aPL in SLE patients. More than $70 \%$ in both groups were well informed on the increased rate of fetal and maternal complications in both SLE and APS.

For fetal surveillance, $87 \%$ and $90 \%$ of obstetricians preformed first and second trimester U/S, and $79 \%$ preformed second trimester Doppler.

For contraception, $(57.7 \%$ vs $56.7 \%$ ) discuss contraceptive choices with their patients. The majority considered it safe to use IUDs $(73.9 \%$ vs $76 \%)$ and condoms ( 84.7 vs $85.4 \%$ ) in both SLE and APS patients. On the other hand, for hormonal contraception- Levonorgestrol IUD, Depoprovera, COCP, and POP- only $14.6 \%, 22.9 \%, 26.1 \%, 24.8 \%$ of rheumatologists and $18.5 \%, 27.2 \%, 29.9 \%, 26.8 \%$ of obstetricians considered them unsafe to use in APS.

Concerning treatment, the majority considered low dose presnidone to be safe during pregnancy $(94.8 \%$ vs $80 \%)$ and breastfeeding $(87.6 \%$ vs $64.3 \%)$. The majority also agreed on avoidance of Methotrexate $(94.8 \%$ vs $84.1 \%)$ and Cyclophosphamide $(89.7 \%$ vs $66.2 \%)$. However, regarding Hydroxychloroquine and Azathioprine use in pregnancy there was a significant discrepancy between rheumatologists and obstetricians, $(89.7 \%$ vs $42 \%)$ and $(78.4 \%$ vs $36.9 \%)$ believed them safe to use in pregnancy. For Mycophenolate Mofetil, $(80.4 \%$ vs $46.5 \%)$ said that it should be avoided in pregnancy. Regarding ART ( $45.4 \%$ vs $71 \%$ ) considered it safe to use in stable SLE/APS

Conclusion: The gaps in knowledge identified include the use of hormonal contraception in APS patients and the proper utilization of important medications to prevent and treat lupus flares. Initiation of shared Rheumatology/ obstetric clinics and focusing on the identified educational topics, would lessen the gap in knowledge and discrepancies in practice improve overall patient management. References:

[1] Andreoli L et al. Ann Rheum Dis. 2017;76(3):476-85.

Disclosure of Interests: None declared

DOI: 10.1136/annrheumdis-2020-eular.2162

\section{$\mathrm{AB} 1147$ \\ SCREENED - HIGH REMISSION RATES UNDERLINE THE BENEFIT OF SCREENING CONSULTATION MODELS FOR EARLY RECOGNITION AND TREATMENT OF RHEUMATIC AND MUSCULOSKELETAL DISEASES}

K. Benesova ${ }^{1}$, V. Lion ${ }^{1}$, O. Hansen ${ }^{1}$, H. M. Lorenz ${ }^{1}{ }^{1}$ University Hospital Heidelberg, Department of Medicine V, Hematology, Oncology and Rheumatology, Heidelberg, Germany

Background: Early recognition and treatment of rheumatic and musculoskeletal diseases (RMDs) is of critical importance for the individual outcome. However nationwide health care structures in Germany do not facilitate early access to initial rheumatologic evaluation. Furthermore, waiting times of several months due to substantial capacity constrains in regional rheumatology care services compromise the prognostically relevant "window of opportunity" for subsequent sustained remission. To promote early detection of RMDs, the Division of Rheumatology at the University hospital Heidelberg, Germany has launched a unique screening consultation model that offers early access to rheumatologic evaluation on regional level.

Objectives: The registry-based study SCREENED ("Screen for early diagnosis") has been initiated to monitor the outcome of patients that were diagnosed with an RMD at the screening clinic and to assess the costs and benefits of this consultation model for the regional quality of care. 
Methods: The screening consultation model has been launched in two phases: in the first phase $(02 / 2016$ - 01/2018), a screening clinic open to all patients without previous rheumatologic evaluation with appointments according to the registration order has been established through rearrangement of available capacities. In contrast to regular appointments, the screening clinic took place in shorter consultation time frames and without additional diagnostic procedures. In the second phase (02/2018 - 01/2020), in order to manage and prioritize access to rheumatologic care at our division more efficiently, prior to appointment allocation (not only) to the screening clinic all new patient registrations became subject to a preselection procedure based on the evaluation of an anamnesis questionnaire, medical reports and laboratory findings by an experienced rheumatologist. Furthermore, SCREENED project has been launched for scientific evaluation of both phases of the consultation model.

Results: The screening consultation model achieved a significant reduction in waiting times to few weeks compared to six months for a regular appointment. In the first phase, the screening clinic had a high sensitivity of $94.3 \%$ and an improvable specificity of $31.1 \%$. In the retrospective cohort, high remission rates have been observed over all RMD entities $(120 / 206=58.3 \%$ patients based on physicians' assessment in the follow-up after screening clinic) and in rheumatoid arthritis (RA) in particular $(38 / 61=62.3 \%$ and $33 / 55=60 \%$ patients with DAS28 score $<2,6$ after 12 and 24 months respectively). Remission was usually reached within a year after the first appointment $(9.5 \pm 6.7$ months), however, a trend to higher remission rates in patients with shorter illness duration was obvious. In RA patients, csDMARDs have been initiated in a third of patients immediately at diagnosis in the screening clinic and in another third within six months after the first appointment. After 12 months, > $80 \%$ have received csDMARDs, while only $14.2 \%$ needed b/tsDMARDs in the follow-up over 24 months.

Conclusion: High sensitivity and significant reduction in waiting times for initial rheumatologic evaluation in the screening clinic pave the way for early recognition and treatment of RMDs. Subsequently, high remission rates in the follow-up were reached. In RA, a high proportion of patients only required csDMARDs to achieve sustained remission. A correspondingly small proportion of patients necessitating b/tsDMARD in the follow-up points towards a significant health economic benefit of the early rheumatologic intervention in the screening consultation model.

Disclosure of Interests: Karolina Benesova Grant/research support from: Study grants for SCREENED study by Abbvie, Novartis and Rheumaliga Baden-Württemberg, Consultant of: One-time participation in Novartis advisory board., Vivienne Lion Grant/research support from: Grant/research support from: Study grants for SCREENED study by Abbvie, Novartis and Rheumaliga Baden-Württemberg, Oliver Hansen Grant/research support from: Grant/ research support from: Study grants for SCREENED study by Abbvie, Novartis and Rheumaliga Baden-Württemberg, Hanns-Martin Lorenz Grant/research support from: Consultancy and/or speaker fees and/or travel reimbursements: Abbvie, MSD, BMS, Pfizer, Celgene, Medac, GSK, Roche, Chugai, Novartis, UCB, Janssen-Cilag, Astra-Zeneca, Lilly. Scientific support and/or educational seminars and/or clinical studies: Abbvie, MSD, BMS, Pfizer, Celgene, Medac, GSK, Roche, Chugai, Novartis, UCB, Janssen-Cilag, Astra-Zeneca, Lilly, Baxter, SOBI, Biogen, Actelion, Bayer Vital, Shire, Octapharm, Sanofi, Hexal, Mundipharm, Thermo Fisher., Consultant of: see above

DOI: 10.1136/annrheumdis-2020-eular.4887

\section{AB1148 ECONOMIC BENEFIT FROM IMPROVEMENTS IN HEALTH-RELATED QUALITY OF LIFE WITH UPADACITINIB AND COMPARISONS WITH TOFACITINIB AND METHOTREXATE IN PATIENTS WITH MODERATELY TO SEVERELY ACTIVE RHEUMATOID ARTHRITIS}

A. Bensimon ${ }^{1}$, M. Yang ${ }^{1}$, E. Orvis ${ }^{1}$, J. Clewell ${ }^{2}$, N. Tundia ${ }^{2} .{ }^{1}$ Analysis Group, Inc., Boston, United States of America; ${ }^{2}$ AbbVie Inc., North Chicago, United States of America

Background: Rheumatoid arthritis (RA) is a chronic, debilitating autoimmune disease and is associated with high direct medical costs. Treatment of RA with disease-modifying anti-rheumatic drugs (DMARDs) can improve patients' health-related quality of life (HRQOL) and has the potential to reduce direct medical costs associated with RA. Treatment with janus kinase inhibitors, such as upadacitinib (UPA), has shown improvements in HRQOL in patients with RA [1].
Objectives: To estimate the economic benefit from improvements in HRQOL and to compare estimated direct medical costs between: (1) UPA and tofacitinib (TOFA) and (2) UPA monotherapy and methotrexate (MTX) monotherapy in patients with RA.

Methods: This economic analysis used individual patient-level data from 2 randomized clinical trials (RCTs) of UPA (SELECT-NEXT and SELECTMONO) and published aggregate data from 1 RCT of TOFA (ORAL-Standard) in patients with moderate to severe RA that collected repeated measurements of HRQOL based on the Short Form 36 Health Survey (SF-36). Estimated direct medical costs per patient per month (PPPM) for UPA 15mg once daily (QD) and MTX were estimated based on observed SF-36 Physical (PCS) and Mental Component Summary (MCS) scores in the SELECT RCTs using a published regression algorithm [2]. Direct medical costs PPPM for TOFA 5mg twice daily (BID) were estimated from Rendas-Baum, et al [3], which applied the same regression algorithm to SF-36 PCS and MCS scores observed in the ORAL-Standard RCT. Resulting estimates of direct medical costs PPPM in the short-term (12-14 weeks) and long-term (48 weeks) were compared between UPA and TOFA and between UPA and MTX. Costs were inflation-adjusted to 2018 US dollars. Bootstrapping was used to generate $95 \%$ confidence intervals $(\mathrm{Cl})$

Results: Over 12 weeks, estimated direct medical costs PPPM were \$186 lower (95\% Cl: \$21, \$364) in patients treated with UPA compared with those treated with TOFA. Estimated long-term medical costs PPPM at Weeks 24 and 48 (Figure 1) and cumulative costs over the entire 48-week period (difference: $\$ 1,452 ; 95 \% \mathrm{Cl}$ : $\$ 906, \$ 2,086$; Table) were significantly lower for UPA than for TOFA. Over 14 weeks, estimated direct medical costs PPPM were $\$ 370$ lower (95\% Cl: \$147, \$575) in patients treated with UPA monotherapy compared with those treated with MTX alone. Estimated long-term direct medical costs at Week 48 (Figure 2) and cumulative costs over the entire 48-week period (difference: $\$ 2,120 ; 95 \% \mathrm{Cl}$ : $\$ 1,398, \$ 2,861$; Table) were significantly lower for UPA monotherapy compared with MTX alone.

Conclusion: Based on improvements in $\mathrm{HRQOL}$ in the short-term and longterm, UPA $15 \mathrm{mg}$ QD was associated with significantly lower direct medical costs than TOFA $5 \mathrm{mg}$ BID in patients with active RA. UPA 15mg QD monotherapy was associated with significantly lower direct medical costs than MTX monotherapy in patients with active RA. These results provide evidence of the economic benefits of UPA as a novel treatment for moderate to severe RA.

References:

[1] Strand V, et al. Arthritis Res Ther 2019;21:272;

[2] Fleishman JA, et al. Med Care 2006;44(Suppl 5):154-63;

[3] Rendas-Baum R, et al. Rheumatology 2017;56:1386-94.

Table. Cumulative cost savings over 48 weeks with UPA vs TOFA and UPA vs MTX

\begin{tabular}{ccc}
\hline Treatment & Total 48-week medical costs $(\$)$ & Difference $(95 \% \mathrm{Cl})$ \\
\hline UPA vs TOFA & & \\
TOFA 5mg BID & 8,964 & $1,452(906,2,086)$ \\
UPA 15mg QD & 7,511 & - \\
UPA vs MTX & & $2,120(1,398,2,861)$ \\
MTX & 9,833 & - \\
UPA 15mg QD & 7,713 & \\
\hline
\end{tabular}

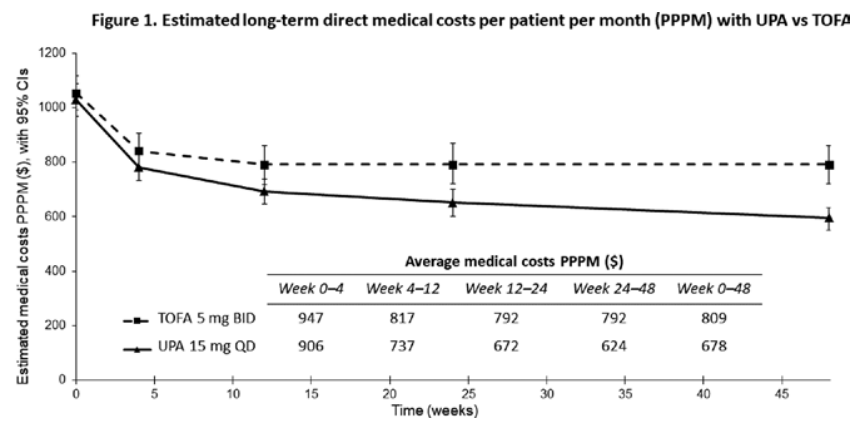

